\title{
INFINITE METACYCLIC GROUPS AND THEIR NON-ABELIAN TENSOR SQUARES
}

\author{
JAMES R. BEUERLE ${ }^{1}$ AND LUISE-CHARLOTTE KAPPE ${ }^{2}$ \\ ${ }^{1}$ Department of Mathematics, Elon College, \\ Elon College, NC 27244, USA (jbeuerle@elon.edu) \\ ${ }^{2}$ Department of Mathematical Sciences, SUNY at Binghamton, \\ Binghamton, NY 13902-6000, USA (menger@math.binghamton.edu)
}

(Received 7 January 1999)

\begin{abstract}
In this paper we classify all infinite metacyclic groups up to isomorphism and determine their non-abelian tensor squares. As an application we compute various other functors, among them are the exterior square, the symmetric product, and the second homology group for these groups. We show that an infinite non-abelian metacyclic group is capable if and only if it is isomorphic to the infinite dihedral group.
\end{abstract}

Keywords: non-abelian tensor products; metacyclic groups; capability; homological functors

AMS 1991 Mathematics subject classification: Primary 20F05

\section{Introduction}

For any group $G$, the non-abelian tensor square $G \otimes G$ is generated by the symbols $g \otimes h$, subject to the relations

$$
g g^{\prime} \otimes h=\left({ }^{g} g^{\prime} \otimes{ }^{g} h\right)(g \otimes h) \quad \text { and } \quad g \otimes h h^{\prime}=(g \otimes h)\left({ }^{h} g \otimes{ }^{h} h^{\prime}\right),
$$

where $g, g^{\prime}, h, h^{\prime}$ range independently over $G$ and ${ }^{g} h=g h g^{-1}$. It is a special case of the non-abelian tensor product of groups which has its roots in algebraic $K$-theory as well as topology. For Brown and Loday in [2] and [3], the non-abelian tensor product is the direct outgrowth of their involvement with generalized Van Kampen theorems. Earlier work by Miller [13] and Dennis [4] is in context with Schur multipliers and algebraic $K$-theory, respectively. Independently, Lue [12] defines the non-abelian tensor products in the setting of nilpotent groups. In [1], Brown, Johnson and Robertson started the investigation of non-abelian tensor squares as group theoretic objects. One of their main results is the explicit computation of non-abelian tensor squares. For a complete overview of the non-abelian tensor squares that have been computed so far, we refer to [10].

The topic of this paper is the classification of infinite metacyclic groups up to isomorphism and the determination of their non-abelian tensor squares. As an application we 
compute various functors derived from the non-abelian squares of these groups, among them the exterior square, the symmetric product, the Schur multiplier and other homotopy groups. In addition, we characterize infinite metacyclic groups which are capable in the sense of [8], i.e. central quotients of a group.

A metacyclic group is the extension of a cyclic group by a cyclic group. In our classification of infinite metacyclic groups, it comes as no surprise that all such groups are split extensions. However, the breakdown into isomorphism classes is different than one would expect from the finite case (see, for example, Exercise 6 on p. 186 of [5]).

Based on earlier results in [1], Johnson already determined the non-abelian tensor square of a finite split metacyclic group in [9]. We want to mention here that in a future publication, J.R.B. will address the non-abelian tensor square of a finite non-split metacyclic group, thus completing the determination of non-abelian tensor squares of metacyclic groups.

The commutator subgroup of a metacyclic group is cyclic. As a consequence of this, its non-abelian tensor square is abelian (see Proposition 3.5 in [14]). This fact helps us in using the concept of a crossed pairing in our computations. We define it here in the case relevant for non-abelian tensor squares. For the general case of the non-abelian tensor product we refer to $[\mathbf{1}]$.

Definition 1.1 (see [1]). Let $G$ and $L$ be groups. A function $\Gamma: G \times G \rightarrow L$ is called a crossed pairing if the following relations hold for all $g, g^{\prime}, h, h^{\prime} \in G$ :

$$
\begin{aligned}
& \Gamma\left(g g^{\prime}, h\right)=\Gamma\left({ }^{g} g^{\prime},{ }^{g} h\right) \cdot \Gamma(g, h) \\
& \Gamma\left(g, h h^{\prime}\right)=\Gamma(g, h) \cdot \Gamma\left({ }^{h} g,{ }^{h} h^{\prime}\right) .
\end{aligned}
$$

Crossed pairings allow us to determine homomorphic images of $G \otimes G$ as follows.

Proposition 1.2 (see [1]). A crossed pairing $\Gamma$ determines a unique homomorphism of groups $\Gamma^{*}: G \otimes G \rightarrow L$, such that $\Gamma^{*}(g \otimes h)=\Gamma(g, h)$ for all $g, h \in G$.

For obvious reasons we only determine the non-abelian tensor squares of infinite metacyclic groups with non-trivial commutator subgroup. Contrary to the case of nilpotent groups of class 2 and rank 2, not all of our groups are homomorphic images of a universal parent group. For all infinite metacyclic groups whose commutator subgroup is finite or which are the extension of an infinite cyclic group by an infinite cyclic group, we have to apply directly crossed pairings to obtain their non-abelian tensor squares (Theorem 4.3). However, infinite metacyclic groups with infinite commutator subgroup are all homomorphic images of one group, namely the extension of an infinite cyclic group by an infinite cyclic group. The following result then allows us to obtain the tensor square of such a group as a homomorphic image of the tensor square of the universal parent group of this class (Theorem 4.4).

Theorem 1.3 (see [11]). Let $G, H, K, L$ be groups with $\pi: H \rightarrow G$ an epimorphism, $\psi: K \rightarrow L$ a homomorphism, and $\Gamma: H \times H \rightarrow K$ a crossed pairing. If $\Gamma$ (ker $\pi, H)$ and $\Gamma(H, \operatorname{ker} \pi)$ are contained in $\operatorname{ker} \psi$, then there exists a crossed pairing $\Delta: G \times G \rightarrow L$ 
for which the following diagram commutes:

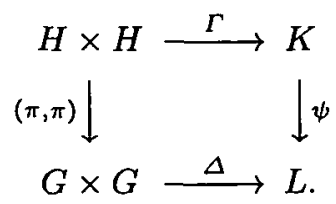

The relations of the non-abelian tensor square with other well-known constructions is one of the motivations to compute it. After that, the desired constructions can be obtained with little effort.

There exists a homomorphism $\kappa: G \otimes G \rightarrow G^{\prime}$ sending $g \otimes h$ to $[g, h]$. We write $J(G)$ for the kernel of $\kappa$. Its topological interest is the formula $J(G) \cong \pi_{3} S K(G, 1)$, as given in [2] and [3], where $S K(G, 1)$ is the suspension of $K(G, 1)$. The group $J(G)$ lies in the centre of $G \otimes G$.

Following the notation and terminology in [7], let $\Delta(G)$ denote the subgroup of $J(G)$ generated by the elements $(x \otimes y)(y \otimes x)$ for $x, y \in G$. The symmetric product of $G$ is then defined as $G \tilde{\otimes} G=(G \otimes G) / \Delta(G)$. We set $\tilde{J}(G)=J(G) / \Delta(G)$. It is shown in [3] that $\tilde{J}(G) \cong \pi_{4} S^{2} K(G, 1)=\pi_{2}^{s} K(G, 1)$.

Let $\nabla(G)$ denote the subgroup of $J(G)$ generated by the elements $x \otimes x$ for $x \in G$. The exterior square of $G$ is then defined as $G \wedge G=(G \otimes G) / \nabla(G)$. We set $J(G) / \nabla(G)=$ $M(G)$, which is otherwise known as the Schur multiplier of $G$. It has been shown in [13] that $M(G) \cong H_{2}(G)$, the second homology group of $G$.

We summarize these results in the following theorem, which (modulo different notation and terminology) can already be found in [3].

Theorem 1.4 (see [3]). Let $G$ be a group. Then the rows are exact in the following commutative diagram

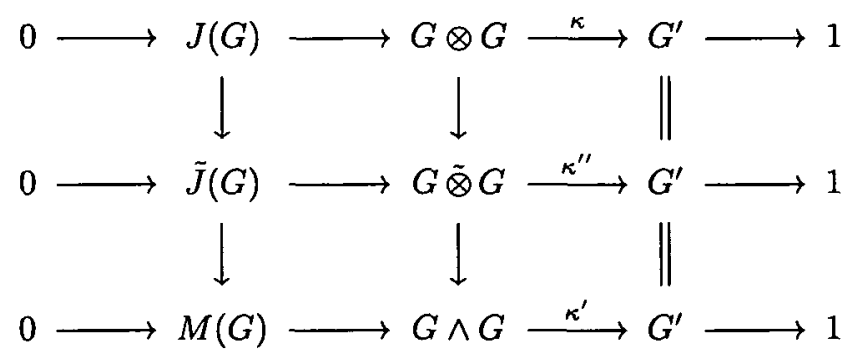

where $\kappa(g \otimes h)=\kappa^{\prime \prime}(g \tilde{\otimes} h)=\kappa^{\prime}(g \wedge h)=[g, h]$.

In our last section, we will compute $G \tilde{\otimes} G, G \wedge G$ as well as $J(G), \tilde{J}(G)$ and $M(G)$ for an infinite metacyclic group $G$.

Following [8], we say that a group is capable if there exists a group $H$ such that $G \cong H / Z(H)$. In [6], a necessary and sufficient condition is given for a group to be capable involving the exterior centre of the group, defined as

$$
Z^{\wedge}(G)=\{g \in G \mid g \wedge x=1 \wedge \text { for all } x \in G\}
$$


Theorem 1.5 (see [6]). A group is capable if and only if its exterior centre is trivial.

In the final theorem of this paper we will show that the only capable non-abelian infinite metacyclic group is the infinite dihedral group.

\section{Some preparatory results}

This section contains some results to be used in the next three sections. First, we define a number theoretic function and study its properties.

We start with some notation. Let $m$ be a non-negative integer. For $m>0$, let $C_{m}$ denote the cyclic group of order $m, \mathbb{Z}_{m}$ the ring of integers modulo $m$, and $\mathcal{U}_{m}$ the set of integers relatively prime to $m$. If $m=0$, we denote by $C_{0}$ the infinite cyclic group, $\mathbb{Z}_{0}$ or $\mathbb{Z}$ the ring of integers, and $\mathcal{U}_{0}=\{1,-1\}$, the group of units in $\mathbb{Z}$. Furthermore, for $m>0$ denote by $r^{-1}$ the smallest positive integer in $\mathcal{U}_{m}$ such that $r r^{-1} \equiv 1 \bmod m$, whenever $r \in \mathcal{U}_{m}$. Let $x, y$ be non-negative integers. For $x$ and $y$ not both zero, let $(x, y)=\operatorname{gcd}(x, y)$ and define $(0,0)=0$. The function in the following definition, suggested by Guzmán, will be used extensively in subsequent sections. It considerably facilitates our exposition.

Definition 2.1. Let $m, r$ be integers with $m \geqslant 0$ and $r \in \mathcal{U}_{m}$. Then define a function $E_{m}: \mathcal{U}_{m} \times \mathbb{Z} \rightarrow \mathbb{Z}$ by

$$
E_{m}(r, x)=\left\{\begin{array}{lll}
x & \text { if } r \equiv 1 \quad \bmod m \text { or } x=0, \\
1+r+\cdots+r^{x-1} & \text { if } r \neq 1 \quad \bmod m \text { and } x>0, \\
-r^{x} E_{m}(r,-x) & \text { if } r \not \equiv 1 \quad \bmod m \text { and } x<0 .
\end{array}\right.
$$

In the following three lemmas, detailed information on the function $E_{m}$ is provided facilitating the verification that a certain mapping in Theorem 4.3 is a crossed pairing. The proof of the first lemma will be given in $\S 3$.

Lemma 2.2. Let $m, r, x, y$ be integers with $m \geqslant 0$ and $r \in \mathcal{U}_{m}$. Then

$$
\begin{gathered}
E_{m}(r, x+y) \equiv E_{m}(r, x)+r^{x} E_{m}(r, y) \quad \bmod m ; \\
\left(1-r^{x}\right) E_{m}(r, y) \equiv\left(1-r^{y}\right) E_{m}(r, x) \quad \bmod m .
\end{gathered}
$$

Lemma 2.3. Let $m, r, x$ be integers with $m \geqslant 0$ and even, and $r \in \mathcal{U}_{m}$. Then

$$
E_{m}(r, x) \equiv x \bmod 2 .
$$

Proof. If $r \equiv 1 \bmod m$ or $x=0$, there is nothing to show. Suppose now $r \not \equiv 1$ $\bmod m$. Since $m$ is even, it follows that $r$ is odd. Let $x>0$. Then, by the definition of $E_{m}, E_{m}(r, x)$ is the sum of an even number of odd integers or a sum of an odd number of odd integers, depending on whether $x$ is even or odd, respectively, and our claim follows. Now let $x<0$. Observing that $r^{x}$ is also odd, we conclude that our claim is true in this case as well.

Lemma 2.4. Let $m, r, x$ be integers with $m \geqslant 0$ and even, and $r \in \mathcal{U}_{m}$. Then

$$
\begin{aligned}
r^{2 x} \equiv 1 & & \bmod (m, 2(r-1)) \\
r^{x}+(r-1) x \equiv 1 & & \bmod (m, 2(r-1)) .
\end{aligned}
$$


Proof. If $r \equiv 1 \bmod m$ or $x=0$, the result is obvious. So, let $r \not \equiv 1 \bmod m$. By (2.3), we obtain $r^{2 x} \equiv\left(r^{2 x}-1\right)+1 \equiv(r-1) E_{m}(r, 2 x)+1 \bmod m$, with $y=1$ and $x$ replaced with $2 x$. Now, Lemma 2.3 implies $E_{m}(r, 2 x) \equiv 0 \bmod 2$. We conclude that $r^{2 x} \equiv 1 \bmod (m, 2(r-1))$. We observe that $(2.5)$ is an immediate consequence of (2.4).

For easier reference, we include a lemma on finitely generated abelian groups.

Lemma 2.5. Let $A=\left\langle a_{1}, \ldots, a_{n}\right\rangle$ be a finitely generated abelian group, and $B=$ $\left\langle b_{1}\right\rangle \times \cdots \times\left\langle b_{n}\right\rangle$ a direct sum of $n$ cyclic groups such that the order of $a_{i}$ divides the order of $b_{i}$ for $i=1, \ldots, n$. If $\phi: A \rightarrow B$ is a homomorphism such that $\phi\left(a_{i}\right)=b_{i}$, then $\phi$ is an isomorphism.

\section{The classification of infinite metacyclic groups}

In this section we classify infinite metacyclic groups up to isomorphism. We start with a lemma which provides us with formulae to compute products, conjugates and powers of elements in a split metacyclic group.

Lemma 3.1. Let $m, n, r$ be integers with $m, n$ non-negative and $r \in \mathcal{U}_{m}$, and let

$$
G=\left\langle a, b \mid a^{m}=b^{n}=1,[a, b]=a^{1-r}\right\rangle,
$$

a split metacyclic group. Then every $g \in G$ can be written as $g=a^{\alpha} b^{\beta}$, where $\alpha$ and $\beta$ are integers which are unique modulo $m$ and $n$, respectively.

If $h \in G$ with $h=a^{\gamma} b^{\delta}$, then we have the following multiplication, conjugation and power formulae in $G$ :

$$
\begin{aligned}
g h & =a^{\alpha+\gamma r^{\delta}} b^{\beta+\delta} \\
{ }^{g} h & =a^{\gamma\left(1-r^{\beta}\right)+\alpha r^{\gamma}} b^{\beta} ; \\
g^{\sigma} & =a^{\alpha E_{m}\left(r^{\beta}, \sigma\right)} b^{\beta \sigma} \quad \text { for any integer } \sigma .
\end{aligned}
$$

Proof. First we observe that $b a=a^{r} b$ and, as an immediate consequence, $b^{-1} a=$ $a^{r^{-1}} b^{-1}$. The verification of (3.1) and (3.2) now follows immediately. To avoid the use of non-trivial identities for $E_{m}$, we use induction on both positive and negative $\sigma$. For positive $\sigma$, (3.3) follows by induction. For negative $\sigma$, we observe that $g^{-1}=a^{-\alpha r^{-\beta}} y^{-\beta}$ and proceed by induction.

Using Lemma 3.1, Lemma 2.2 can be now be proved without the need for any cases.

Proof of Lemma 2.2. Let $m, r, x, y$ be integers with $m$ non-negative and $r \in \mathcal{U}_{m}$. Furthermore, let $G=\left\langle a, b \mid a^{m}=b^{n}=1,[a, b]=a^{1-r}\right\rangle$. From (3.1) and (3.3) we get $a^{E_{m}(r, x+y)} b^{x+y}=(a b)^{x+y}=(a b)^{x}(a b)^{y}=a^{E_{m}(r, x)} b^{x} a^{E_{m}(r, y)} b^{y}=a^{E_{m}(r, x)+r^{x} E_{m}(r, y)} b^{x+y}$. Thus, $E_{m}(r, x+y) \equiv E_{m}(r, x)+r^{x} E_{m}(r, y) \bmod m$. For (2.3), we observe that $E_{m}(r, x)+$ $r^{x} E_{m}(r, y) \equiv E_{m}(r, x+y) \equiv E_{m}(r, y)+r^{y} E_{m}(r, x) \bmod m$, which yields the desired result. 
Theorem 3.2. Let $G$ be an infinite metacyclic group. Then $G$ is isomorphic to

$$
G(m, n, r)=\left\langle a, b \mid a^{m}=b^{n}=1,[a, b]=a^{1-r}\right\rangle,
$$

where $m, n, r$ are non-negative integers, with $m n=0$ and $r \in \mathcal{U}_{m}$. Moreover, if $r \equiv-1$ mod $m$, then $n$ is even, or if $r \equiv 1 \bmod m$, then $m=0$. In particular, $G(m, n, r) \cong$ $G\left(m^{\prime}, n^{\prime}, s\right)$ if and only if $m=m^{\prime}, n=n^{\prime}$ and $r \equiv s^{l} \bmod m$, where $l=1,-1$.

Proof. The proof of the first part of our claim is routine and will be omitted. To prove the second part, assume $G(m, n, r) \cong G\left(m^{\prime}, n^{\prime}, s\right)$. It can easily be seen that this implies $m=m^{\prime}$ and $n=n^{\prime}$. However, we will show explicitly that this also implies $r \equiv s^{l}$ mod $m$, where $l=1,-1$, since this is in contrast to what holds in the finite case, as already mentioned in $\S 1$.

If $m=0$, then $r=s=1$ when the groups are abelian, and $r=s=-1$ otherwise. Thus, our claim holds trivially in this case.

Now assume $m>0$, and thus $n=0$. For ease of computation, let $G(m, n, r)=\langle a, b\rangle$ and $G\left(m^{\prime}, n^{\prime}, s\right)=\langle c, d\rangle$. Denoting the isomorphism from $G(m, 0, r)$ to $G\left(m^{\prime}, 0, s\right)$ by $\psi$, there exist integers $i, j$ and $l$ such that $\psi(a)=c^{i}$ and $\psi(b)=c^{j} d^{l}$. Furthermore, $b a=a^{r} b$, implies $c^{j} d^{l} c^{i}=\left(c^{i}\right)^{r} c^{j} d^{l}$. From (3.1) and (3.3) we obtain $c^{j+i s^{l}} d^{l}=c^{i r+j} d^{l}$. This yields $i s^{l} \equiv i r \bmod m$. Since $\psi$ is injective and $\psi(a)=c^{i}$, it follows that $(i, m)=1$. So

$$
s^{l} \equiv r \bmod m \text {. }
$$

Also, since $\psi$ is surjective, there are integers $u$ and $v$ such that $d=\psi\left(a^{u} b^{v}\right)$. By the definition of $\psi$ and (3.3), we obtain

$$
d=\psi\left(a^{u} b^{v}\right)=c^{i u}\left(c^{j} d^{l}\right)^{v}=c^{i u+j E_{m}\left(s^{l}, v\right)} d^{l v} .
$$

So, since $d$ has infinite order, it follows that $l v=1$. This, together with (3.4), yields $r \equiv s^{l} \bmod m$, with $l=1,-1$, the desired result.

It is clear that the reverse implication holds.

\section{The non-abelian tensor square of an infinite metacyclic group}

Our first proposition contains an expansion formula for $g \otimes h$, where $g$ and $h$ are elements in an infinite metacyclic group.

Proposition 4.1. Let $G$ be a group of type $G(m, n, r)$, as given in Theorem 3.2, with $g, h \in G$, where $g=a^{\alpha} b^{\beta}$ and $h=a^{\gamma} b^{\delta}$ with $\alpha, \beta, \gamma, \delta$ integers. Then

$$
g \otimes h=(a \otimes a)^{\lambda}(a \otimes b)^{\mu}(b \otimes a)^{\nu}(b \otimes b)^{\rho}
$$

with

$$
\begin{aligned}
& \lambda \equiv \equiv \gamma+E_{m}(-1, m+1)(r-1) \\
& \quad \times\left[\beta \gamma(\alpha-1)+\alpha \delta(\gamma-1)+\frac{1}{2}\left(\beta \gamma^{2}+\delta \alpha^{2}+\mu+\nu\right)\right] \bmod m \\
& \mu \equiv \alpha E_{m}(r, \delta) \quad \bmod m \\
& \nu \equiv \gamma E_{m}(r, \beta) \quad \bmod m \\
& \rho \equiv \beta \delta \bmod n
\end{aligned}
$$


Proof. Direct calculations yield $(a \otimes a)^{m}=(a \otimes b)^{m}=(b \otimes a)^{m}=(b \otimes b)^{n}=1_{\otimes}$. So (4.1) is well defined. The formula follows by repeatedly applying the defining relations for $G(m, n, r)$, as given in Theorem 3.2, and the defining relations of the tensor square to $a^{\alpha} b^{\beta} \otimes a^{\gamma} b^{\delta}$ and collecting terms, observing that $G \otimes G$ is abelian by Proposition 3.5 in [11]. We observe that $E_{m}(-1, m+1)=0$ or 1 , if $m$ is odd or even, respectively. In particular, $\lambda=\alpha \gamma$ for odd $m$. If $m$ is even, then $r-1$ is even, so $\frac{1}{2}(r-1)$ is defined.

The next proposition is, in part, a consequence of the preceding one.

Proposition 4.2. Let $G$ be a non-abelian group of type $G(m, n, r)$. Then

$$
G \otimes G=\langle a \otimes a,(a \otimes b)(b \otimes a), b \otimes a, b \otimes b\rangle .
$$

For the orders of the generators the following estimates hold:

$$
\begin{aligned}
& (a \otimes a)^{(m, n, 2(r-1))}=1_{\otimes}, \quad(b \otimes a)^{m}=1_{\otimes}, \\
& {[(a \otimes b)(b \otimes a)]^{(m, r-1)}=1_{\otimes}, \quad(b \otimes b)^{n}=1_{\otimes} .}
\end{aligned}
$$

Proof. The first claim, that $G \otimes G$ is generated by the elements above, is an immediate consequence of Proposition 4.1. It remains to establish the order bounds. The bounds $(a \otimes a)^{m}=(a \otimes b)^{m}=(b \otimes a)^{m}=(b \otimes b)^{n}=1_{\otimes}$ were already obtained in Proposition 4.1. We now compute a better upper bound for the order of $a \otimes a$. Applying (4.1) and (11) of Proposition 3 in [1], we obtain $(a \otimes a)^{(1-r)^{2}}=a^{1-r} \otimes a^{1-r}=[a, b] \otimes[a, b]=$ $[a \otimes b, a \otimes b]=1_{\otimes}$. Observing that $b$ acts trivially on $a \otimes a$ (see Proposition 4 of $[1]$ ), it follows that $a \otimes a={ }^{b}(a \otimes a)=a^{r} \otimes a^{r}=(a \otimes a)^{r^{2}}$, hence $(a \otimes a)^{r^{2}-1}=1_{\otimes}$. Since $\left(r^{2}-1\right)-(r-1)^{2}=2(r-1)$, we obtain $(a \otimes a)^{2(r-1)}=1_{\otimes}$. So, $(a \otimes a)^{(m, 2(r-1))}=1_{\otimes}$. If $n=0$, then $(m, 2(r-1))=(m, 0,2(r-1))$, and our result follows. Let $n>0, m=0$, and, hence, $r=-1$ and $n$ is even. From (4.1) we get $1_{\otimes}=a \otimes b^{n}=(a \otimes a)^{n}$. This, together with the above, yields $(a \otimes a)^{(n, 2(r-1))}=1_{\otimes}$. Next, consider $(a \otimes b)(b \otimes a)$. By $(4.1)$ and the defining relations of $G$, it follows that $[(a \otimes b)(b \otimes a)]^{1-r}=\left(a^{1-r} \otimes b\right)\left(b \otimes a^{1-r}\right)=$ $([a, b] \otimes b)(b \otimes[a, b])$. Using (9) and (10) of Proposition 3 in [1], we obtain

$$
([a, b] \otimes b)(b \otimes[a, b])=(a \otimes b) \cdot\left({ }^{b}(a \otimes b)^{-1}\right) \cdot{ }^{b}(a \otimes b) \cdot(a \otimes b)^{-1}=1_{\otimes} .
$$

Hence, $[(a \otimes b)(b \otimes a)]^{1-r}=1_{\otimes}$. For the other bound, note that $[(a \otimes b)(b \otimes a)]^{m}=(a \otimes$ $b)^{m}(b \otimes a)^{m}=1_{\otimes}$. This, together with the above, yields $((a \otimes b)(b \otimes a))^{(m, r-1)}=1_{\otimes}$.

We are now in a position to determine the non-abelian tensor square of an infinite metacyclic group in the case $n=0$.

Theorem 4.3. Let $G$ be a group of type $G(m, 0, r)$. Then

$$
G \otimes G \cong C_{(m, 2(r-1))} \times C_{(m, r-1)} \times C_{m} \times C_{0}
$$

with generators $a \otimes a,(a \otimes b)(b \otimes a), b \otimes a, b \otimes b$, respectively. 
Proof. Set $L=\left\langle u_{1}\right\rangle \times\left\langle u_{2}\right\rangle \times\left\langle u_{3}\right\rangle \times\left\langle u_{4}\right\rangle$, where $\left\langle u_{1}\right\rangle=C_{(m, 2(r-1))},\left\langle u_{2}\right\rangle=C_{(m, r-1)}$, $\left\langle u_{3}\right\rangle=C_{m}$, and $\left\langle u_{4}\right\rangle=C_{0}$. We represent the elements $l \in L$ as 4-tuples, listing the exponents of generators as coordinates as follows:

$$
l=\left(\exp \left(u_{1}\right), \exp \left(u_{2}\right), \exp \left(u_{3}\right), \exp \left(u_{4}\right)\right)
$$

and denote the identity element of $L$ by $\overline{0}$. Set $g=a^{\alpha} b^{\beta}, h=a^{\gamma} b^{\delta}$ and $g^{\prime}=h^{\prime}=a^{\alpha^{\prime}} b^{\beta^{\prime}}$, where $\beta, \delta, \beta^{\prime}$ are integers and $\alpha, \gamma, \alpha^{\prime}$ are integers modulo $m$.

Define a map $\psi: G \times G \rightarrow L$ as

$$
\psi(g, h)=(\lambda, \mu, \nu-\mu, \rho)
$$

where $\lambda, \mu, \nu, \rho$ are defined as in (4.1). Since $\beta, \delta$ are unique integers and $\alpha, \gamma$ are unique integers modulo $m$, the mapping $\psi: G \times G \rightarrow L$ given by (4.5) is well defined.

Next we will show that $\psi$ is a crossed pairing, i.e. (1.1) and (1.2) are satisfied. Using (4.5), (3.1) and (3.3), we obtain

$$
\psi\left(g g^{\prime}, h\right)-\psi\left({ }^{g} g^{\prime},{ }^{g} h^{\prime}\right)-\psi(g, h)=\left(z_{1}, z_{2}, z_{3}, z_{4}\right)
$$

where

$$
\begin{aligned}
z_{1} \equiv( & \left.+\alpha^{\prime} r^{\beta}\right) \gamma+(r-1) E_{m}(-1, m+1)\left[\left(\beta^{\prime}+\beta\right) \gamma\left(\alpha+\alpha^{\prime} r^{\beta}-1\right)\right. \\
& +\left(\alpha+\alpha^{\prime} r^{\beta}\right) \delta(\gamma-1)+\frac{1}{2}\left(\left(\beta^{\prime}+\beta\right) \gamma^{2}+\delta\left(\alpha+\alpha^{\prime} r^{\beta}\right)^{2}+\left(\alpha+\alpha^{\prime} r^{\beta}\right) E_{m}(r, \delta)\right. \\
& \left.\left.+\gamma E_{m}\left(r, \beta^{\prime}+\beta\right)\right)\right]-\left[\left(\alpha(1-r) E_{m}\left(r, \beta^{\prime}\right)+\alpha^{\prime} r^{\beta}\right)\left(\alpha(1-r) E_{m}(r, \delta)+\gamma r^{\beta}\right)\right. \\
& +(r-1) E_{m}(-1, m+1)\left[\beta^{\prime} \gamma\left(\alpha^{\prime}-1\right)+\delta \alpha^{\prime}(\gamma-1)\right. \\
& \left.\left.+\frac{1}{2}\left(\beta^{\prime} \gamma^{2}+\delta \alpha^{\prime 2}+\alpha^{\prime} r^{\beta} E_{m}(r, \delta)+\gamma r^{\beta} E_{m}\left(r, \beta^{\prime}\right)\right)\right]\right] \\
& \quad-\left[\alpha \gamma+(1-r) E_{m}(-1, m+1)\right] \beta \gamma(\alpha-1)+\alpha \delta(\gamma-1) \\
& \left.\left.+\frac{1}{2}\left(\beta \gamma^{2}+\delta \alpha^{2}+\alpha E_{m}(r, \delta)+\gamma E_{m}(r, \beta)\right)\right]\right] \bmod (m, 2(r-1)), \\
z_{2} \equiv(\alpha & \left.+\alpha^{\prime} r^{\beta}\right) E_{m}(r, \delta)-\left[\left(\alpha(1-r) E_{m}\left(r, \beta^{\prime}\right)+\alpha^{\prime} r^{\beta}\right) E_{m}(r, \delta)\right] \\
& \quad-\alpha E_{m}(r, \delta) \bmod (m, r-1), \\
z_{3} \equiv \gamma & E_{m}\left(r, \beta+\beta^{\prime}\right)-\left[\alpha(1-r) E_{m}(r, \delta)+\gamma r^{\beta}\right] E_{m}\left(r, \beta^{\prime}\right) \\
& \quad-\gamma E_{m}(r, \beta)-\left[\left(\alpha+\alpha^{\prime} r^{\beta}\right) E_{m}(r, \delta)\right. \\
& \left.\quad-\left[\left(\alpha(1-r) E_{m}\left(r, \beta^{\prime}\right)+\alpha^{\prime} r^{\beta}\right) E_{m}(r, \delta)\right]-\alpha E_{m}(r, \delta)\right] \bmod m, \\
z_{4}=0 &
\end{aligned}
$$

By Lemma 2.2, it follows that $z_{1} \equiv 0 \bmod (m, 2(r-1))$ and $z_{3} \equiv 0 \bmod m$. Direct computation yields $z_{2} \equiv 0 \bmod (m, r-1)$. Hence, (4.6) becomes

$$
\psi\left(g g^{\prime}, h\right)-\psi\left({ }^{g} g^{\prime},{ }^{g} h^{\prime}\right)-\psi(g, h)=\overline{0}
$$

and (1.1) holds. To verify (1.2), we exploit the 'symmetry' of $\psi$, i.e. if $\psi(g, h)=(\lambda, \mu, \nu-$ $\mu, \rho)$, then $\psi(h, g)=(\lambda, \nu, \mu-\nu, \rho)$. Thus, (1.2) is satisfied and $\psi$ is a crossed pairing. By 
Proposition 1.2 it follows that there is a homomorphism $\psi^{*}$ of $G \otimes G$ into $L$. In particular, we have $\psi^{*}(a \otimes a)=(1,0,0,0), \psi^{*}((a \otimes b)(b \otimes a))=(0,1,0,0), \psi^{*}(b \otimes a)=(0,0,1,0)$, and $\psi^{*}(b \otimes b)=(0,0,0,1)$.

Thus, the generators of $G \otimes G$ map to the generators of $L$. By the order estimates given in Proposition 4.2, the orders of the generators of $G \otimes G$ divide the corresponding orders of the generators of $L$. By Lemma $2.5, \psi^{*}$ is an isomorphism. We conclude that $G \otimes G \cong C_{(m, 2(r-1))} \times C_{(m, r-1)} \times C_{m} \times C_{0}$.

The groups of the form $G(0, n,-1)$, where $n>0$ and even, are homomorphic images of $G(0,0,-1)$. This helps us in the determination of their non-abelian tensor square, which is the content of the last theorem of this section.

Theorem 4.4. Let $G$ be a group of type $G(0, n,-1)$. Then

$$
G \otimes G \cong C_{(n, 4)} \times C_{2} \times C_{0} \times C_{n}
$$

with generators $a \otimes a,(a \otimes b)(b \otimes a), b \otimes a, b \otimes b$, respectively.

Proof. Let $G=G(0, n,-1)$ and $H=G(0,0,-1)$. For ease of computation set $G=$ $\langle a, b\rangle$ and $H=\langle c, d\rangle$. Let $\pi: H \rightarrow G$ be the homomorphism given by $\pi(c)=a$ and $\pi(d)=b$. Observe that $\operatorname{ker} \pi=\left\langle d^{n}\right\rangle$. Set $K=C_{4} \times C_{2} \times C_{0} \times C_{0}$, with generators $x_{1}$, $x_{2}, x_{3}, x_{4}$, respectively, and observe that $K \cong H \otimes H$ by Theorem 4.3. Let $\psi: H \times H \rightarrow$ $K$ be the crossed pairing defined in (4.5). Furthermore, let $L=C_{(n, 4)} \times C_{2} \times C_{0} \times$ $C_{n}$, with generators $y_{1}, y_{2}, y_{3}, y_{4}$, respectively. Define a homomorphism $\phi: K \rightarrow L$, by $\phi\left(x_{i}\right)=y_{i}$. In order to apply Proposition 1.3 , we have to verify that $\phi(\psi(\operatorname{ker} \pi, H))=$ $\phi(\psi(H, \operatorname{ker} \pi))=0$. For $\left(d^{\tau n}, c^{\gamma} d^{\delta}\right) \in \operatorname{ker} \pi \times H$ with $\tau \in \mathbb{Z}$, we have

$$
\phi\left(\psi\left(d^{n \tau}, c^{\gamma} d^{\delta}\right)\right)=\phi(n \tau \gamma(\gamma-2), 0,0, n \tau \delta)=(0,0,0,0)
$$

So, $\phi(\psi(\operatorname{ker} \pi, H))=0$. Similarly, $\phi(\psi(H, \operatorname{ker} \pi))=0$. Thus, by Proposition 1.3, there exists a crossed pairing $\Delta: G \times G \rightarrow L$ such that the diagram commutes. Hence, by Proposition 1.2, the crossed pairing $\Delta$ lifts to a homomorphism $\Delta^{*}: G \otimes G \rightarrow L$ with $\Delta^{*}(g \otimes h)=\Delta(g, h)$. In particular, $\Delta^{*}(a \otimes a)=(1,0,0,0), \Delta^{*}((a \otimes b)(b \otimes a))=(0,1,0,0)$, $\Delta^{*}(b \otimes a)=(0,0,1,0)$, and $\Delta^{*}(b \otimes b)=(0,0,0,1)$.

Hence, the generators of $G \otimes G$ are mapped to generators of $L$. By the order estimates given in Proposition 4.2, the orders of the generators of $G \otimes G$ divide the corresponding orders of the generators of $L$. By Proposition 2.5, it follows that $\Delta^{*}$ is an isomorphism. Thus, $G \otimes G \cong C_{(n, 4)} \times C_{2} \times C_{0} \times C_{n}$.

It is observed that the finite split metacyclic groups are homomorphic images of $G(m, 0, r)$. A similar technique that was used to compute the non-abelian tensor square of groups of type $G(0, n,-1)$ can be used to obtain Johnson's result in [9] for finite split metacyclic groups. 


\section{Applications}

In this section we compute various functors that arise out of the non-abelian tensor square as homomorphic images or kernels of homomorphisms for the class of infinite metacyclic groups. In addition, we determine all non-abelian infinite metacyclic groups which are capable.

We first determine the exterior square and the second homology group.

Theorem 5.1. Let $G$ be a non-abelian infinite metacyclic group of type $G(m, n, r)$. Then

$$
\begin{gathered}
G \wedge G \cong C_{m} ; \\
H_{2}(G) \cong \begin{cases}C_{(m, r-1)} & \text { if } m>0, \\
1 & \text { if } m=0 .\end{cases}
\end{gathered}
$$

Proof. Let $G$ be a non-abelian infinite metacyclic group of type $G(m, n, r)$. Using Proposition 4.1, we obtain $\nabla(G)=\langle a \otimes a,(a \otimes b)(b \otimes a), b \otimes b\rangle$. From Theorems 4.3 and 4.4 we conclude that $G \wedge G=G \otimes G / \nabla(G) \cong\langle b \otimes a\rangle \cong C_{m}$, proving (5.1).

To show (5.2), suppose first $m>0$. By direct calculations, it follows that $\left|G^{\prime}\right|=$ $(m /(m, r-1))$. From (5.1), we have that $G \wedge G=C_{m}$. So, by Theorem 1.4 and the above, it follows that $H_{2}(G)$ is cyclic of order $(m, r-1)$. Now suppose $m=0$. Since $\kappa^{\prime}(b \wedge a)=a^{-2}$ and $G^{\prime}=\left\langle a^{2}\right\rangle$ has infinite order, we conclude that $H_{2}(G)=1$ in this case.

Next, we determine the third homotopy group of the suspension of $K(G, 1)$.

Theorem 5.2. Let $G$ be a non-abelian infinite metacyclic group of type $G(m, n, r)$. Then

$$
\pi_{3} S K(G, 1) \cong \begin{cases}C_{(m, 2(r-1))} \times C_{(m, r-1)} \times C_{(m, r-1)} \times C_{0} & \text { if } m>0, \\ C_{(n, 4)} \times C_{2} \times C_{n} & \text { if } m=0 .\end{cases}
$$

Proof. Let $G$ be a non-abelian infinite metacyclic group of type $G(m, n, r)$. Then, from Theorem 1.4 and the remark preceding it, we have $J(G) \cong \pi_{3} S K(G, 1)$. It follows from the definition of $\kappa$, that $\langle a \otimes a,(a \otimes b)(b \otimes a), b \otimes b\rangle \leqslant J(G)$. To finish the computation of $J(G)$, it suffices to compute the kernel of $\kappa$ when restricted to the cyclic subgroup $\langle b \otimes a\rangle$. First, suppose $m=0$ and, hence, $r=-1$. Since $\kappa(b \otimes a)=a^{-2}$ is a generator of $G^{\prime}$ and $G^{\prime}$ is infinite cyclic, we obtain $J(G)=\langle a \otimes a,(a \otimes b)(b \otimes a), b \otimes b\rangle$. Hence, $J(G) \cong C_{(n, 4)} \times C_{2} \times C_{n}$, when $m=0$. Now, let $m>0$. In this case, we have $|b \otimes a|=m$ and $\left|G^{\prime}\right|=(m /(m, r-1))$. Thus,

$$
J(G)=\left\langle a \otimes a,(a \otimes b)(b \otimes a),(b \otimes a)^{(m /(m, r-1))}, b \otimes b\right\rangle .
$$

Hence, $J(G) \cong C_{(m, 2(r-1))} \times C_{(m, r-1)} \times C_{(m, r-1)} \times C_{0}$, for the case $m>0$.

Now we determine the symmetric product and the corresponding kernel of the commutator map. 
Theorem 5.3. Let $G$ be a non-abelian infinite metacyclic group of type $G(m, n, r)$. Then, with generators $a \tilde{\otimes} a, b \tilde{\otimes} a, b \tilde{\otimes} b$, respectively,

$$
\begin{aligned}
& G \tilde{\otimes} G \cong \begin{cases}C_{(m, 2)} \times C_{m} \times C_{2} & \text { if } m>0, \\
C_{2} \times C_{0} \times C_{2} & \text { if } m=0 ;\end{cases} \\
& \pi_{2}^{s} K(G, 1) \cong \begin{cases}C_{(m, 2)} \times C_{(m, r-1)} \times C_{2} & \text { if } m>0, \\
C_{2} \times C_{2} & \text { if } m=0 .\end{cases}
\end{aligned}
$$

Proof. Let $G$ be a non-abelian infinite metacyclic group of type $G(m, n, r)$. From (4.1) we have $\Delta(G)=\left\langle(a \otimes a)^{2},(a \otimes b)(b \otimes a),(b \otimes b)^{2}\right\rangle$. The desired result now follows from Theorem 4.3 for $m>0$ and Theorem 4.4 if $m=0$ and the fact that $n$ is even for $m=0$.

To prove (5.4), we observe that $\pi_{2}^{s} K(G, 1) \cong \tilde{J}(G)$. From the definition of $\kappa^{\prime \prime}$, we have $\langle a \tilde{\otimes} a, b \tilde{\otimes} b\rangle \leqslant \tilde{J}(G)$. To compute $\tilde{J}(G)$, we determine which power of $b \tilde{\otimes} a$ is in $\tilde{J}(G)$. First, suppose $m=0$ and, hence, $n$ is even. Since $\kappa^{\prime \prime}(b \tilde{\otimes} a)=a^{-2}$ is a generator of $G^{\prime}$ and $G^{\prime}$ is infinite cyclic, we obtain $\tilde{J}(G)=\langle a \tilde{\otimes} a, b \tilde{\otimes} b\rangle$. Hence, $\tilde{J}(G) \cong C_{2} \times C_{2}$, when $m=0$. Now, suppose $m>0$. By (5.3) we have $|b \tilde{\otimes} a|=m$. Since $\kappa^{\prime \prime}(b \tilde{\otimes} a)=a^{-2}$, and $G^{\prime}=\left\langle a^{2}\right\rangle$, we conclude that

$$
\tilde{J}(G)=\left\langle a \tilde{\otimes} a,(b \tilde{\otimes} a)^{(m /(m, r-1))}, b \tilde{\otimes} b\right\rangle .
$$

Hence, $\tilde{J}(G) \cong C_{(m, 2)} \times C_{(m, r-1)} \times C_{2}$, for $m>0$.

In our final theorem we determine all capable non-abelian infinite metacyclic groups.

Theorem 5.4. The only non-abelian infinite metacyclic group that is capable is $C_{0} \rtimes$ $C_{2}$, the infinite dihedral group. In particular, if $H=C_{0} \rtimes C_{4}$, then $C_{0} \rtimes C_{2} \cong H / Z(H)$.

Proof. Let $G$ be a non-abelian infinite metacyclic group. Then $G$ is a group of type $G(m, n, r)$. Suppose $g=a^{\alpha} b^{\beta} \in Z^{\wedge}(G)$. Then, from (4.1), we obtain $1_{\wedge}=a^{\alpha} b^{\beta} \wedge b=$ $(a \wedge b)^{\alpha E(r, 1)}=(a \wedge b)^{\alpha}$. Since $|a \wedge b|=m$, we have $a^{\alpha}=1_{G}$. Hence, $Z^{\wedge}(G) \leqslant\langle b\rangle$. To show that $Z^{\wedge}(G)$ is non-trivial and, hence, by Theorem $1.5, G$ is not capable, it suffices to show that there exists $\beta \in \mathbb{N}$, such that $b^{\beta} \wedge a=1_{\wedge}$ and $\beta \neq 0 \bmod n$, since $b^{\beta} \wedge a^{\gamma} b^{\delta}=\left(b^{\beta} \wedge a\right)^{\gamma}$.

First, suppose $m>0$. We have in this case that $n=0$. Since $(r, m)=1$, there is a positive integer, $x$, such that $E(r, x) \equiv 0 \bmod m$. Since $n=0$ we have $b^{x} \neq 1_{G}$, but $b^{x} \wedge a=(b \wedge a)^{E(r, x)}=1 \wedge$. Hence $G$ is not capable if $m>0$.

Next, suppose $m=0$ and, hence, $r=-1$ and $n$ is even. Note that

$$
b^{\beta} \wedge a=(b \wedge a)^{E(-1, \beta)}=1_{\wedge}
$$

if and only if $\beta$ is even. So, if $n \geqslant 4$, then $G$ is not capable, since $1_{G} \neq b^{2} \in Z^{\wedge}(G)$ in this case. If $n=2$, then $G$ is capable. Thus, the only non-abelian infinite metacyclic group that is capable is $C_{0} \rtimes C_{2}$. Let $H=\left\langle c, d \mid d^{4}=1,{ }^{d} c=c^{-1}\right\rangle$. Then, it follows that $Z(H)=\left\langle d^{2}\right\rangle$. The result now follows. 


\section{References}

1. R. BRown, D. L. Johnson AND E. F. Robertson, Some computations of non-abelian tensor products of groups, J. Algebra 111 (1987), 177-202.

2. R. BRown AND J.-L. Loday, Excision homotopique en basse dimension, C. R. Acad. Sci. (Series I) Math. Paris 298 (1984), 353-356.

3. R. BROWN AND J.-L. LODAY, Van Kampen theorems for diagrams of spaces, Topology 26 (1987), 311-335.

4. R. K. DENNIS, In search of new 'homology' functors having a close relationship to $K$ theory (Preprint, Cornell University, Ithaca, NY, 1976).

5. D. S. Dummit And R. M. Foote, Abstract algebra (Prentice Hall, Englewood Cliffs, NJ, 1991).

6. G. ElLIS, Tensor products and $q$-crossed modules, J. Lond. Math. Soc. (2) 51 (1995), 243-258.

7. G. Ellis, On the computation of certain homological functors, J. Comput. Math. 1 (1998), 25-41.

8. M. HALl AND J. K. Senior, The groups of order $2^{n}, n \leqslant 6$ (Macmillan, New York, 1964).

9. D. L. Johnson, The nonabelian tensor square of a finite split metacyclic group, Proc. Edinb. Math. Soc. 30 (1987), 91-96.

10. L.-C. KAPPE, Nonabelian tensor products of groups: the commutator connection, Proc. Groups-St. Andrews 1997 at Bath, London Mathematical Society Lecture Notes, vol. 261 (1999), pp. 187-209.

11. L.-C. KAPPE, N. SARmin AND M. Visscher, Two-generator 2-groups of class 2 and their nonabelian tensor squares, Glasgow Math. J. 41 (1999), 417-430.

12. A. S.-T. LuE, The Ganea map for nilpotent groups, J. Lond. Math. Soc. 14 (1976), 309-312.

13. C. MILleR, The second homology group of a group: relations among commutators, Proc. Am. Math. Soc. 3 (1952), 588-595.

14. M. VISSCHER, On the nilpotency class and solvability length of nonabelian tensor products of groups, Arch. Math. 73 (1999), 161-171. 